\title{
Cytoskeletal and Phosphoinositide Requirements for Muscarinic Receptor Signaling to Focal Adhesion Kinase and Paxillin
}

\author{
Daniel A. Linseman, *Edward L. McEwen, Scott D. Sorensen, and *Stephen K. Fisher \\ Department of Pharmacology and *Neuroscience Laboratory, Mental Health Research Institute, \\ University of Michigan, Ann Arbor, Michigan, U.S.A.
}

\begin{abstract}
The mechanism whereby agonist occupancy of muscarinic cholinergic receptors elicits an increased tyrosine phosphorylation of focal adhesion kinase (FAK) and paxillin has been examined. Addition of oxotremorine-M to $\mathrm{SH}-\mathrm{SY} 5 \mathrm{Y}$ neuroblastoma cells resulted in rapid increases in the phosphorylation of $F A K\left(t_{1 / 2}=2 \mathrm{~min}\right)$ and paxillin that were independent of integrin-extracellular matrix interactions, cell attachment, and the production of phosphoinositide-derived second messengers. In contrast, the increased tyrosine phosphorylations of FAK and paxillin were inhibited by inclusion of either cytochalasin $\mathrm{D}$ or mevastatin, agents that disrupt the cytoskeleton. Furthermore, phosphorylation of FAK and paxillin could be prevented by addition of either wortmannin or LY294002, under conditions in which the synthesis of phosphatidylinositol 4-phosphate was markedly attenuated. These results indicate that muscarinic receptor-mediated increases in the tyrosine phosphorylation of FAK and paxillin in SH-SY5Y neuroblastoma cells depend on both the maintenance of an actin cytoskeleton and the ability of these cells to synthesize phosphoinositides. Key Words: Actin polymerization-Phosphatidylinositol 4phosphate-Cytochalasin - LY-294002 - Mevastatin Wortmannin
\end{abstract}

J. Neurochem. 70, 940-950 (1998).

Focal adhesion kinase (FAK) is a $125-\mathrm{kDa}$ cytoplasmic protein tyrosine kinase originally identified in v-src-transformed chicken embryo fibroblasts (Schaller et al., 1992). Integrin-mediated attachment of fibroblasts to extracellular matrix (ECM) proteins induces the tyrosine phosphorylation of FAK, increased kinase activity, and localization to focal adhesions (Burridge et al., 1992; Guan and Shalloway, 1992; Hanks et al., 1992; Kornberg et al., 1992). Several putative downstream substrates for FAK have been identified, one of which is the cytoskeletal protein paxillin (Bellis et al., 1995; Schaller and Parsons, 1995). Thus, FAK has been implicated as a potential regulator of integrin-mediated signal transduction and cytoskeletal assembly at sites of developing focal adhe- sions. Recently, FAK has been identified in neural tissue both in vitro (Zhang et al., 1994; Derkinderen et al., 1996) and in vivo (Grant et al., 1995). Furthermore, both FAK and paxillin have been implicated in a signal transduction pathway linked to neurite outgrowth (Bianchi et al., 1995; Maroney et al., 1995; Leventhal and Feldman, 1996; Leventhal et al., 1997).

In addition to integrin activation, the tyrosine phosphorylation of FAK is enhanced in fibroblasts in response to multiple stimuli, including transformation by retroviral oncogenes (Guan and Shalloway, 1992), growth factors for receptor tyrosine kinases (Rankin et al., 1996), bioactive lipids (Seufferlein and Rozengurt, $1994 a, b$ ), and phorbol esters (Sinnett-Smith et al., 1993 ). Furthermore, agonist occupancy of receptors that couple to phosphoinositide hydrolysis has also been demonstrated to elicit an increase in the tyrosine phosphorylation of FAK, although the mechanism whereby this occurs has yet to be defined (Sinnett-

Received July 29, 1997; revised manuscript received October 6 , 1997; accepted October 7, 1997.

Address correspondence and reprint requests to Dr. S. K. Fisher at Neuroscience Laboratory, Mental Health Research Institute, University of Michigan, 1103 East Huron Street, Ann Arbor, MI 481041687, U.S.A.

Abbreviations used: $\mathrm{AACOCF}_{3}$, arachidonyl trifluoromethyl ketone; DAG, $s n$-1,2-diacylglycerol; DMEM, Dulbecco's modified Eagle's medium; ECL, enhanced chemiluminescence; ECM, extracellular matrix; FAK, focal adhesion kinase; FCS, fetal calf serum $\mathrm{IP}_{3}$, inositol 1,4,5-trisphosphate; LPA, lysophosphatidic acid; LY-294002, 2-(4-morpholinyl)-8-phenyl-4H-1-benzopyran-4-one; $\mathrm{mAChR}$, muscarinic cholinergic receptor; MAPK, mitogen-activated protein kinase; MLCK, myosin light chain kinase; $\left[{ }^{3} \mathrm{H}\right] \mathrm{NMS}$, $\left[N\right.$-methyl- $\left.{ }^{3} \mathrm{H}\right]$ scopolamine methyl chloride; Oxo-M, N,N,N-trimethyl-4-(2-oxo-1-pyrrolidinyl)-2-butyn-1-ammonium iodide or oxotremorine-M; PA, phosphatidic acid; PAGE, polyacrylamide gel electrophoresis; PBS, phosphate-buffered saline; PBS-T, phosphatebuffered saline ( $\mathrm{pH} 7.4$ ) containing $0.1 \%$ Tween 20 ; PI, phosphatidylinositol; PIC, phosphoinositide-specific phospholipase C; $\mathrm{PI}(4) \mathrm{P}$, phosphatidylinositol 4-phosphate; $\mathrm{PI}(4,5) \mathrm{P}_{2}$, phosphatidylinositol 4,5-bisphosphate; PKC, protein kinase C; PMA, phorbol 12myristate 13-acetate; PVDF, polyvinylidene difluoride; SDS, sodium dodecyl sulfate; TCA, trichloroacetic acid. 
Smith et al., 1993; Leeb-Lundberg et al., 1994; Rankin et al., 1994).

Recently it was reported that activation of muscarinic cholinergic receptors ( $\mathrm{mAChRs}$ ) present on $\mathrm{SH}-$ SY5Y neuroblastoma cells resulted in morphological changes characteristic of neurite outgrowth (Rösner et al., 1995). Given that (a) mAChRs on these cells are known to couple to phosphoinositide hydrolysis (Fisher et al., 1994; Slowiejko et al., 1996) and (b) the presence of both FAK and paxillin has been documented in SH-SY5Y cells (Leventhal and Feldman, 1996; Leventhal et al., 1997), the observations of Rösner et al. ( 1995) prompted us to determine whether the activation of mAChRs in SH-SY5Y cells resulted in an increased tyrosine phosphorylation of FAK and, if so, the mechanism by which this occurs.

The results indicate that, on agonist occupancy of mAChRs in SH-SY5Y cells, there is a rapid increase in the tyrosine phosphorylation of FAK and paxillin. This appears to occur independently of the production of phosphoinositide-derived second messengers but is absolutely dependent on the presence of an intact actin cytoskeleton. Agonist-stimulated tyrosine phosphorylation of both FAK and paxillin was also blocked by pretreatment of the cells with either wortmannin or LY-294002 [2-(4-morpholinyl)-8-phenyl-4H-1-benzopyran-4-one ], under conditions in which the synthesis of phosphatidylinositol (PI) 4-phosphate [PI(4)P] was markedly attenuated. The data suggest that the maintenance of an actin microfilament network and the continuous ability of these cells to synthesize phosphoinositides are prerequisites for the tyrosine phosphorylation of FAK and paxillin that occurs in response to $\mathrm{mAChR}$ activation. A preliminary account of part of this study has previously been reported (Linseman et al., 1997).

\section{MATERIALS AND METHODS}

\section{Materials}

Cytochalasin D, thapsigargin, phorbol 12-myristate 13acetate (PMA), Ro 31-8220, ML-7, arachidonyl trifluoromethyl ketone $\left(\mathrm{AACOCF}_{3}\right)$, quinacrine, 1-oleoyl-2-acetyl$s n$-glycerol, and LY-294002 were obtained from Calbiochem (San Diego, CA, U.S.A.). Atropine, pilocarpine, mevastatin, mevalonic acid lactone, retinoic acid, and wortmannin were from Sigma Chemical Co. (St. Louis, MO, U.S.A.). Oxotremorine-M [Oxo-M; $N, N, N$-trimethyl-4-(2-oxo-1-pyrrolidinyl)-2-butyn-1-ammonium iodide] was purchased from Research Biochemicals International (Natick, MA, U.S.A.). Recombinant C3 exoenzyme was a kind gift from Dr. Carol Williams (Guthrie Research Institute, Sayre, PA, U.S.A.) or was purchased from Upstate Biotechnology (Lake Placid, NY, U.S.A.). All other chemicals were of reagent grade. myo- $\left[2{ }^{3} \mathrm{H}\right]$ Inositol, $\left[{ }^{32} \mathrm{P}\right]$ orthophosphoric acid, $[N-$ methyl$\left.{ }^{3} \mathrm{H}\right]$ scopolamine methyl chloride $\left(\left[{ }^{3} \mathrm{H}\right] \mathrm{NMS}\right)$, reagents for enhanced chemiluminescence (ECL), and peroxidase-conjugated sheep anti-mouse IgG were purchased from Amersham Corp. (Arlington Heights, IL, U.S.A.). $\left[{ }^{32}\right.$ P $]$ NAD was obtained from New England Nuclear (Boston, MA, U.S.A.). Monoclonal antibodies to phosphotyrosine and paxillin and a polyclonal antibody to PI 3-kinase were from Upstate Biotechnology or Transduction Laboratories (Lexington, KY, U.S.A.). Polyclonal antibody to the active forms of ERK1 and ERK2 was purchased from Promega (Madison, WI, U.S.A.). Polyclonal antibody to FAK, peroxidase-conjugated goat anti-rabbit IgG, and agarose-conjugated protein A/G were obtained from Santa Cruz Biotechnology (Santa Cruz, CA, U.S.A.). Tissue culture supplies were purchased from Corning Glass Works (Corning, NY, U.S.A.) and Sarstedt (Newton, NC, U.S.A.). Dulbecco's modified Eagle's medium (DMEM) and fetal calf serum (FCS) were obtained from GIBCO (Grand Island, NY, U.S.A.) and BioWhittaker (Walkersville, MD, U.S.A.), respectively. Human SH-SY5Y neuroblastoma cells were obtained from Dr. June Biedler (Sloan-Kettering Institute, New York, NY, U.S.A.).

\section{Cell culture conditions}

Human SH-SY5Y neuroblastoma cells (passages 68-78) were routinely grown in $75-\mathrm{cm}^{2}$ tissue culture flasks containing $20 \mathrm{ml}$ of DMEM supplemented with $10 \%$ FCS. Cells were grown for $7-14$ days at $37^{\circ} \mathrm{C}$ in a humidified atmosphere consisting of $90 \%$ air and $10 \% \mathrm{CO}_{2}$. Cells were retrieved from the flasks by incubation in a modified Pucks $D_{1}$ solution (Honegger and Richelson, 1976). In experiments using attached cells, the cells were subcultured into $35-\mathrm{mm}-$ diameter, six-well culture plates (Becton Dickinson Labware, Lincoln Park, NJ, U.S.A.) for 2-4 days before treatment. In experiments using differentiated cells, SH-SY5Y cells were grown in the presence of $10 \mu M$ retinoic acid for 6 days before treatment, as described by Rösner et al. (1995). All experiments were performed on cells that had reached confluency.

\section{Treatment conditions and immunoprecipitations}

Following removal of the plating medium, confluent 35 mm-diameter cultures of SH-SY5Y cells were washed once with $2 \mathrm{ml}$ of prewarmed $\left(37^{\circ} \mathrm{C}\right)$ treatment buffer containing $138 \mathrm{~m} M \mathrm{NaCl}, 6 \mathrm{~m} M \mathrm{KCl}, 1 \mathrm{~m} M \mathrm{MgCl}_{2}, 1 \mathrm{~m} M \mathrm{CaCl}_{2}, 1$ $\mathrm{m} M \mathrm{Na}_{2} \mathrm{HPO}_{4}, 5 \mathrm{~m} M \mathrm{NaHCO}_{3}, 5.5 \mathrm{~m} M$ glucose, and 20 $\mathrm{m} M$ HEPES (pH 7.4) (Offermanns et al., 1993) and allowed to equilibrate in the incubator in $1 \mathrm{ml}$ of treatment buffer (with or without appropriate pretreatment) for $30 \mathrm{~min}$. Cells were then stimulated by addition of agonist to the pretreatment solution and returned to the incubator for the appropriate duration. Following agonist exposure, the treatment buffer was removed, and cells were rinsed once with $2 \mathrm{ml}$ of ice-cold phosphate-buffered saline (PBS; pH 7.4). Cells were then incubated on ice and scraped into lysis buffer $(200$ $\mu l$ per well) containing $20 \mathrm{~m} M$ HEPES ( $\mathrm{pH} 7.4$ ), $1 \%$ Triton $\mathrm{X}-100,50 \mathrm{~m} M \mathrm{NaCl}, 1 \mathrm{~m} M$ EGTA, $5 \mathrm{~m} M \beta$-glycerophosphate, $30 \mathrm{~m} M$ sodium pyrophosphate, $100 \mu M$ sodium orthovanadate, $1 \mathrm{~m} M$ phenylmethylsulfonyl fluoride, $10 \mu \mathrm{g} / \mathrm{ml}$ aprotinin, and $10 \mu \mathrm{g} / \mathrm{ml}$ leupeptin (Wahl et al., 1988). Cell debris was removed by centrifugation at $12,000 \mathrm{~g}$ for $5 \mathrm{~min}$ at $4^{\circ} \mathrm{C}$, and the protein concentration of the supernatant was determined using a commercially available protein assay kit (Pierce Chemical Co., Rockford, IL, U.S.A.). Aliquots (200 $\mu \mathrm{g}$ ) of detergent-soluble cellular protein were transferred to tubes containing $1 \mu \mathrm{g}$ of either anti-FAK polyclonal antibody or anti-paxillin monoclonal antibody (final volume, $200 \mu \mathrm{l}$ ) and incubated at $4^{\circ} \mathrm{C}$ for $16-20 \mathrm{~h}$ with continuous mixing. Protein A/G-agarose $(20 \mu \mathrm{l})$ was added for an additional 4 $\mathrm{h}$ with mixing. Immune complexes were pelleted by centrifugation and washed three times with ice-cold lysis buffer. The final agarose pellet was resuspended in an equal volume 
of $2 \times$ sodium dodecyl sulfate (SDS) - polyacrylamide gel electrophoresis (PAGE) sample buffer, boiled for $5 \mathrm{~min}$, and electrophoresed through $10 \%$ polyacrylamide gels (Laemmli, 1970). Proteins were transferred to polyvinylidene difluoride (PVDF) membranes (Millipore Corp., Bedford, MA, U.S.A.) and processed for immunoblot analysis. Experiments using SH-SY5Y cells maintained in suspension were performed following detachment of the cells in $D_{1}$ solution. The cells were then washed in prewarmed treatment buffer, resuspended in $5-\mathrm{ml}$ aliquots of treatment buffer (with or without agonist), and incubated in a $37^{\circ} \mathrm{C}$ shaking water bath for $10 \mathrm{~min}$. Samples were agitated every $5 \mathrm{~min}$ to minimize cell aggregation. Following agonist exposure, $20 \mathrm{ml}$ of ice-cold treatment buffer was added, and the samples were immediately placed on ice. Cells were pelleted at $4^{\circ} \mathrm{C}$ by centrifugation at $1,000 \mathrm{~g}$ for $5 \mathrm{~min}$. Following aspiration of the supernatant, the cell pellet was homogenized on ice in $1 \mathrm{ml}$ of lysis buffer by 10 strokes with a glass/glass homogenizer. Cell debris was removed by centrifugation, and immunoprecipitation of either FAK or paxillin was carried out on the supernatant as described above.

\section{Immunoblot analysis}

Nonspecific binding sites were blocked in PBS ( $\mathrm{pH} 7.4$ ) containing $0.1 \%$ Tween 20 (PBS-T) and $1 \%$ bovine serum albumin for $1 \mathrm{~h}$ at room temperature. Primary antibodies were diluted in blocking solution and incubated with the membranes for $1 \mathrm{~h}$. Excess primary antibody was removed by washing the membranes three times in PBS-T. The blots were then incubated in peroxidase-conjugated, secondary antibody diluted in PBS-T for $1 \mathrm{~h}$ and subsequently washed three times in PBS-T. Immunoreactive proteins were detected by ECL. In some experiments, membranes were reprobed after stripping in $0.1 M$ Tris- $\mathrm{HCl}(\mathrm{pH} 8.0), 2 \%$ SDS, and $100 \mathrm{mM} \beta$-mercaptoethanol for $30 \mathrm{~min}$ at $52^{\circ} \mathrm{C}$. The blots were rinsed twice in PBS-T and processed as above with a different primary antibody. Autoluminograms shown are representative of two to four independent experiments. Quantitative analysis of autoluminograms was performed by computer-assisted imaging densitometry (MCID; Imaging Research, St. Catharines, Ontario, Canada).

\section{Measurement of phosphoinositide turnover \\ Confluent dishes of SH-SY5Y cells were prelabeled for $48-72 \mathrm{~h}$ in DMEM/FCS containing $10 \mu \mathrm{Ci} / \mathrm{ml}\left[{ }^{3} \mathrm{H}\right]$ inositol. Following a 10-min pretreatment with either wortmannin or LY-294002, cells were treated with agonist for an additional $10 \mathrm{~min}$, and the accumulation of radiolabeled inositol phos- phates was determined as described previously (Thompson and Fisher, 1990).}

\section{Phospholipid labeling from ${ }^{32} \mathbf{P}_{\mathrm{i}}$}

SH-SY5Y cells plated in 35-mm-diameter wells were incubated at $37^{\circ} \mathrm{C}$ in $1 \mathrm{ml}$ of phosphate-free treatment buffer containing $\sim 20 \mu \mathrm{Ci} / \mathrm{ml}{ }^{32} \mathrm{P}_{\mathrm{i}}$ for $2 \mathrm{~h}$. The labeling solution was aspirated, and unincorporated ${ }^{32} \mathrm{P}_{\mathrm{i}}$ was removed by washing with the same buffer. The cells were then preincubated at $37^{\circ} \mathrm{C}$ for $10 \mathrm{~min}$ with vehicle $(0.2 \%$ dimethyl sulfoxide ), $10 \mu M$ wortmannin, or $100 \mu M$ LY-294002, followed by a further $10-\mathrm{min}$ incubation with either vehicle or $1 \mathrm{~m} M$ Oxo-M. Reactions were terminated by rapid aspiration of the treatment solution followed by addition of $0.5 \mathrm{ml}$ of icecold 5\% trichloroacetic acid (TCA). Cells were scraped into the TCA, transferred to glass tubes with an additional $1 \mathrm{ml}$ of $5 \%$ TCA, and allowed to precipitate on ice for $1 \mathrm{~h}$. Pellets were obtained by low-speed centrifugation at $4^{\circ} \mathrm{C}$ and washed once with $2 \mathrm{ml}$ of ice-cold distilled water, and the final TCA pellet was resuspended in $0.5 \mathrm{ml}$ of water. Lipids were extracted from the pellets with acidified chloroformmethanol as previously described (Fisher and Agranoff, 1980 ). Phospholipids were then separated on oxalate-impregnated TLC plates and quantified according to a previously published method (Fisher et al., 1984).

\section{Other measurements}

PI 3-kinase activity immunoprecipitated from lysates of SH-SY5Y cells was assayed as previously described by Lavie and Agranoff (1996). $\left[{ }^{3} \mathrm{H}\right]$ NMS binding to intact SHSY5Y cells was measured as previously described (Slowiejko et al., 1996). In vitro ADP-ribosylation of Rho proteins in SH-SY5Y lysates by recombinant C3 exoenzyme was performed essentially as described by Yamamoto et al. (1993).

\section{Data analysis}

Results shown represent the mean \pm SEM values for the number $(n)$ of independent experiments performed. Statistical differences between the means of unpaired and paired sets of data were evaluated using Student's two-tailed $t$ tests.

\section{RESULTS}

\section{Agonist occupancy of mAChRs increases the tyrosine phosphorylation of FAK and paxillin}

In preliminary experiments, anti-phosphotyrosine immunoblots of cell lysates from Oxo-M-stimulated SH-SY5Y neuroblastoma cells demonstrated the atropine-sensitive tyrosine phosphorylation of 68- and $125-\mathrm{kDa}$ proteins that comigrated with paxillin and FAK, respectively. To confirm the identity of these tyrosine-phosphorylated proteins, FAK and paxillin were immunoprecipitated from control and agonisttreated SH-SY5Y cell lysates. SH-SY5Y cells treated for $10 \mathrm{~min}$ with $1 \mathrm{~m} M$ Oxo-M showed a $23 \pm 6$-fold increase $(n=9)$ in the tyrosine phosphorylation of FAK (Fig. 1A, upper panel), and a corresponding 6 \pm 1 -fold increase $(n=4)$ in the tyrosine phosphorylatjon of paxillin (Fig. 1B, upper panel). Reprobing of these blots for the precipitated proteins demonstrated equivalent immunoprecipitation efficiencies in the absence or presence of agonist (Fig. 1A and B, lower panels). The agonist-stimulated tyrosine phosphorylation of FAK and paxillin was mediated via mAChRs, because a 10 -min pretreatment with $10 \mu M$ atropine resulted in complete inhibition (Fig. 1A and B, upper panels, respectively). Similar results were also observed for SH-SY 5 Y cells that had been differentiated in the presence of retinoic acid for 6 days before addition of agonist (Fig. 1C and D). Addition of a $1 \mathrm{mM}$ concentration of a partial muscarinic agonist, pilocarpine, also resulted in an increased tyrosine phosphorylation of FAK but of a lesser magnitude than that observed for Oxo-M $(41 \pm 11 \%, \mathrm{n}=4, p<0.05)$.

The time course and dose-response relationships for the Oxo-M-stimulated tyrosine phosphorylation of FAK were also determined. The tyrosine phosphorylation of FAK was half-maximal following a 2-min ex- 


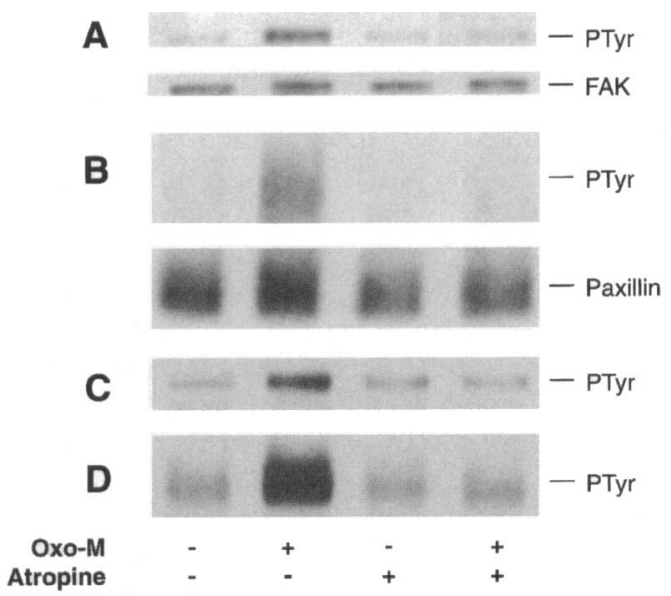

FIG. 1. Oxo-M stimulates the atropine-sensitive tyrosine phosphorylation of FAK and paxillin in SH-SY5Y neuroblastoma cells. Confluent cultures of SH-SY5Y cells were pretreated for $10 \mathrm{~min}$ in either the absence (buffer alone) or presence of $10 \mu \mathrm{M}$ atropine before addition of either buffer or Oxo-M (final concentration, $1 \mathrm{mM}$ ) for an additional $10 \mathrm{~min}$. Following treatment, cells were scraped into lysis buffer, and supernatants containing 200 $\mu \mathrm{g}$ of protein were immunoprecipitated with $1 \mu \mathrm{g}$ of either a polyclonal antibody to FAK (A) or a monoclonal antibody to paxillin (B) as described in Materials and Methods. Immune complexes were subjected to SDS-PAGE, transferred to PVDF membranes, and immunoblotted for either phosphotyrosine (PTyr; upper panels) or the precipitated protein (lower panels). The blots in $A$ and $B$ are representative of four and two independent experiments, respectively. The experiment described above was also performed in SH-SY5Y cells that had been differentiated for 6 days with $10 \mu \mathrm{M}$ retinoic acid before addition of Oxo$M$ with or without atropine, subsequent immunoprecipitation of FAK (C) and paxillin (D), and immunoblotting for PTyr.

posure to Oxo-M, was maximal at $20 \mathrm{~min}$, and returned to approximately the basal level by $120 \mathrm{~min}$ in the continued presence of the agonist (Fig. 2). Oxo-M

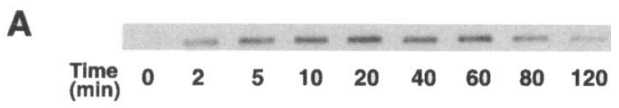

B

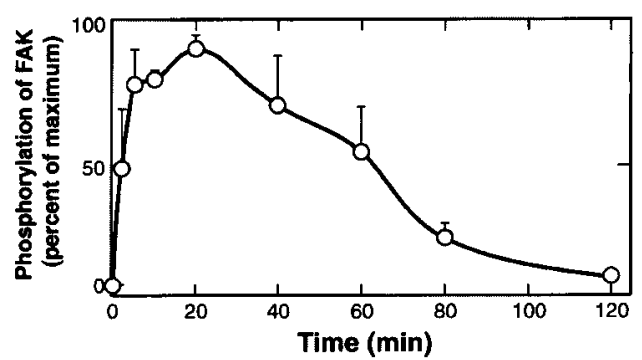

FIG. 2. Time course of Oxo-M-stimulated tyrosine phosphorylation of FAK. A: Confluent cultures of SH-SY5Y cells were treated with $1 \mathrm{mM}$ Oxo-M for various times, and FAK immune complexes were isolated, subjected to SDS-PAGE, transferred to PVDF, and immunoblotted for phosphotyrosine as described in the legend to Fig. 1. B: Densitometric analysis of OxO-M-mediated increases in tyrosine phosphorylation of FAK obtained from three independent experiments. addition stimulated the tyrosine phosphorylation of FAK with an $\mathrm{EC}_{50}$ value of $\sim 10 \mu \mathrm{M}$. In all subsequent experiments, the tyrosine phosphorylation of FAK and paxillin was assessed in undifferentiated SH-SY5Y cells treated with $1 \mathrm{~m} M$ Oxo-M for $10 \mathrm{~min}$.

\section{Oxo-M-stimulated tyrosine phosphorylation of FAK and paxillin is independent of cell attachment}

Previous reports have demonstrated that the coordinated tyrosine phosphorylation of FAK and paxillin can be mediated through interaction of cell surface integrins with ECM proteins (Burridge et al., 1992). Because the present experiments were conducted on SH-SY5Y cells grown on uncoated plastic, the potential for significant involvement of integrin-ECM interactions was minimal. However, we could not exclude the possibility that SH-SY5Y cells may secrete some ECM components in response to agonist stimulation that could interact with cell surface integrins and subsequently elicit the tyrosine phosphorylation of FAK and paxillin via an integrin clustering mechanism. Therefore, the ability of Oxo-M to stimulate the tyrosine phosphorylation of FAK and paxillin in SH-SY5Y cells maintained in suspension was assessed. Detached SH-SY5Y cells responded to the addition of Oxo-M with increases in the tyrosine phosphorylation of FAK (Fig. 3A) and paxillin (Fig. 3B) that were similar in magnitude to those observed for attached cells (see Fig. 1A and 1B). These results suggest that the mAChR-mediated tyrosine phosphorylation of FAK and paxillin is independent of integrin-ECM interactions and cell attachment.

\section{Production of phosphoinositide-derived second messengers is not required for agonist-stimulated FAK phosphorylation}

Activation of mAChRs in SH-SY5Y cells results in the formation of both $s n$-1,2-diacylglycerol (DAG) and inositol 1,4,5-trisphosphate $\left(\mathrm{IP}_{3}\right)$, which subsequently activate protein kinase $\mathrm{C}(\mathrm{PKC})$ and initiate

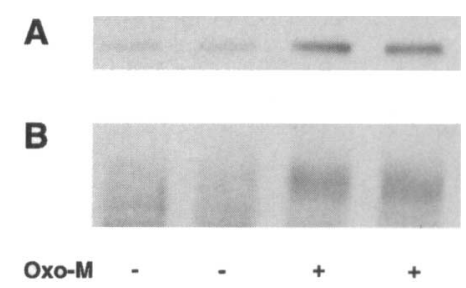

FIG. 3. Oxo-M induces the tyrosine phosphorylation of $F A K$ and paxillin in SH-SY5Y cells maintained in suspension. Confluent flasks of SH-SY5Y cells were detached in $D_{1}$ solution, resuspended in buffer with or without Oxo-M (final concentration, 1 $\mathrm{mM}$ ), and incubated in a shaking water bath at $37^{\circ} \mathrm{C}$ for $10 \mathrm{~min}$. Following treatment, FAK (A) or paxillin (B) immune complexes were isolated, subjected to SDS-PAGE, transferred to PVDF, and immunoblotted for phosphotyrosine as described in the legend to Fig. 1. The blots in A and $B$ are representative of four and two independent experiments, respectively. 
the mobilization of intracellular $\mathrm{Ca}^{2+}$, respectively. Therefore, the role, if any, that these second messengers play in the mediation of the Oxo-M-stimulated tyrosine phosphorylation of FAK was next investigated.

Two approaches were used to inhibit the activation of PKC. First, SH-SY5Y cells were pretreated for 30 min with a $1 \mu M$ concentration of the cell-permeant PKC inhibitor Ro 31-8220 before Oxo-M addition. Ro 31-8220 pretreatment failed to attenuate the Oxo-Mstimulated tyrosine phosphorylation of either FAK (Fig. 4A) or paxillin (data not shown). Second, PKC was down-regulated by preincubating SH-SY5Y cells for $18 \mathrm{~h}$ with a $1 \mu M$ concentration of a tumor-promoting phorbol ester, PMA, before a 10-min incubation with either $1 \mathrm{~m} M$ Oxo-M or $100 \mathrm{n} M$ PMA. In vehiclepretreated cells, both Oxo-M and PMA stimulated the phosphorylation of FAK (Fig. 4B). Thus, PKC activation alone is capable of eliciting FAK phosphorylation in SH-SY5Y cells. When cells were pretreated for 18 h with $1 \mu M$ PMA to down-regulate PKC, the basal level of tyrosine phosphorylation of FAK was increased. Under these conditions, a subsequent acute exposure to PMA did not increase the phosphorylation of FAK above this basal level (Fig. 4B). In contrast, chronic pretreatment of cells with PMA did not impair
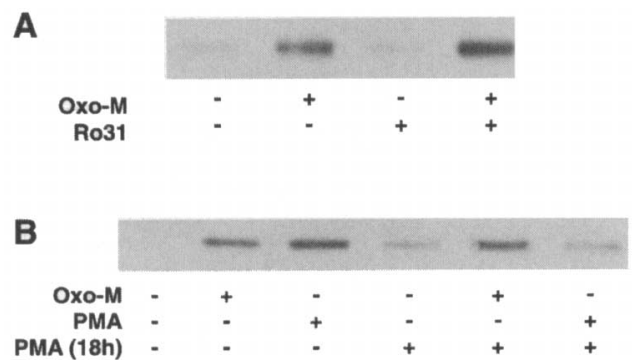

C

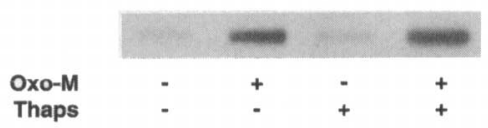

FIG. 4. Muscarinic receptor-mediated phosphorylation of FAK does not require PKC activity or the release of $\mathrm{Ca}^{2+}$ from intracellular stores. A: Confluent cultures of SH-SY5Y cells were pretreated for $30 \mathrm{~min}$ with either vehicle or a $1 \mu \mathrm{M}$ concentration of the PKC inhibitor Ro 31-8220 (Ro31) before addition of either buffer or Oxo-M (final concentration, $1 \mathrm{mM}$ ) for an additional 10 min. Following treatment, FAK immune complexes were isolated, subjected to SDS-PAGE, transferred to PVDF, and immunoblotted for phosphotyrosine as described in the legend to Fig. 1. B: PKC was down-regulated by an 18-h preincubation with $1 \mu M$ PMA, and cells were subsequently incubated in the presence of vehicle, $1 \mathrm{mM}$ Oxo-M, or $100 \mathrm{nM}$ PMA for $10 \mathrm{~min}$. FAK immune complexes were immunoblotted for phosphotyrosine. C: Confluent cultures of SH-SY5Y cells were pretreated for $30 \mathrm{~min}$ with either vehicle or $1 \mu M$ thapsigargin (Thaps) before addition of either buffer or Oxo-M (final concentration, $1 \mathrm{mM}$ ) for an additional $10 \mathrm{~min}$. Following treatment, FAK immune complexes were immunoblotted for phosphotyrosine. The blots in $A$ and $C$ are each representative of three independent experiments. The blot in $B$ is representative of two independent experiments. the ability of Oxo-M to enhance the phosphorylation of FAK (Fig. 4B). These findings suggest that DAG activation of PKC is not involved in mediating the Oxo-M-stimulated tyrosine phosphorylation of FAK.

The potential role of $\mathrm{IP}_{3}$-sensitive calcium release in mediating the tyrosine phosphorylation of FAK and paxillin following $\mathrm{mAChR}$ activation was also assessed. We have previously shown that pretreatment of SH-SY5Y cells for 30 min with $1 \mu M$ thapsigargin totally abolishes the $\mathrm{IP}_{3}$-mediated increase in intracellular calcium concentration stimulated by addition of Oxo-M (Slowiejko et al., 1994). In contrast, thapsigargin pretreatment did not inhibit the Oxo-M-stimulated tyrosine phosphorylation of either FAK (Fig. 4C) or paxillin (data not shown). Furthermore, no reduction in the magnitude of Oxo-M-induced phosphorylation of FAK was observed when SH-SY5Y cells were treated in a modified buffer that lacked calcium but contained $1 \mathrm{mM} \mathrm{NiCl}{ }_{2}$ [conditions that have previously been shown to block the plateau phase of $\mathrm{IP}_{3}$ production and capacitative calcium entry (Wojcikiewicz et al., 1994)]. These results suggest that $\mathrm{IP}_{3^{-}}$ sensitive calcium release and subsequent capacitative calcium entry are not involved in mediating the OxoM-induced tyrosine phosphorylation of FAK and paxillin.

In addition to $\mathrm{DAG}$ and $\mathrm{IP}_{3}$, we also investigated the potential involvement of two other phosphoinositidespecific phospholipase C (PIC)-derived second messengers [lysophosphatidic acid (LPA) and phosphatidic acid (PA)] in mediating the agonist-stimulated tyrosine phosphorylation of FAK. LPA has previously been shown to stimulate FAK phosphorylation in fibroblasts (Seufferlein and Rozengurt, 1994b), and both of these lipids elicit an increase in the tyrosine phosphorylation of FAK when added to SH-SY5Y neuroblastoma cells (data not shown). However, neither the addition of two inhibitors of phospholipase $\mathrm{A}_{2}$ ( $\mathrm{AACOCF}_{3}$ and quinacrine) nor inclusion of 1-oleoyl2-acetyl-sn-glycerol (a competitive substrate for DAG kinase) had any significant effect on the magnitude of Oxo-M-induced tyrosine phosphorylation of FAK.

\section{Role of the cytoskeleton and Rho in $\mathrm{MAChR}$ - mediated FAK and paxillin tyrosine phosphorylation}

Previous studies have demonstrated that the coincident tyrosine phosphorylation of FAK and paxillin mediated by multiple agonists is dependent on the integrity of the cellular actin microfilament network (Seufferlein and Rozengurt, 1994a,b; Flinn and Ridley, 1996). In SH-SY5Y cells, a 30-min pretreatment with $1 \mu M$ cytochalasin D (a compound that selectively disrupts actin microfilaments) completely abolished the Oxo-M-stimulated tyrosine phosphorylation of both FAK (Fig. 5A) and paxillin (Fig. 5B). Cytochalasin D was also effective at blocking the agoniststimulated phosphorylation of FAK in SH-SY5Y cells maintained in suspension (data not shown). In con- 
A

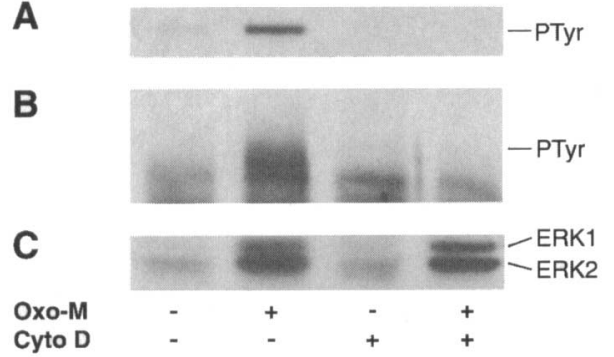

FIG. 5. Cytochalasin $D(C y t o ~ D)$ pretreatment inhibits the OxoM-stimulated tyrosine phosphorylation of FAK and paxillin. Confluent cultures of SH-SY5Y cells were pretreated for $30 \mathrm{~min}$ with either vehicle or $1 \mu M$ Cyto $D$ before addition of either buffer or Oxo-M (final concentration, $1 \mathrm{mM}$ ) for an additional 10 min. Following treatment, FAK (A) or paxillin (B) immune complexes were isolated, subjected to SDS-PAGE, transferred to PVDF, and immunoblotted for phosphotyrosine (PTyr) as described in the legend to Fig. 1. The blots in $A$ and $B$ are representative of three and two independent experiments, respectively. C: $\mathrm{SH}-$ SY5Y cells were pretreated for $30 \mathrm{~min}$ with either vehicle or 10 $\mu M$ Cyto $D$ before agonist addition. Cell lysates were subjected to SDS-PAGE and immunoblotted with an antibody to the active forms of ERK1 and ERK2.

trast, cytochalasin D pretreatment had no effect either on the mAChR-mediated activation of ERK1 and ERK2 (Fig. 5C) or on agonist-induced mAChR sequestration monitored by a loss of $\left[{ }^{3} \mathrm{H}\right] \mathrm{NMS}$ binding sites (an $~ 40 \%$ loss in control cells vs. a $45 \%$ loss in cytochalasin D-pretreated cells).

The agonist-stimulated tyrosine phosphorylation of FAK and paxillin was also significantly attenuated when SH-SY5Y cells were preincubated for $24 \mathrm{~h}$ with $10 \mu M$ mevastatin. Mevastatin is an inhibitor of hydroxymethylglutaryl-CoA reductase, which has been shown to destabilize actin microfilaments via blockade of synthesis of mevalonate, a precursor required for the isoprenylation of low-molecular-weight $G$ proteins involved in cytoskeletal regulation (Bokoch and Prossnitz, 1992; Fenton et al., 1992; Bifulco et al., 1993). Mevastatin pretreatment significantly inhibited the Oxo-M-stimulated tyrosine phosphorylation of both FAK (Fig. 6A) and paxillin (Fig. 6B). Mevastatin also induced marked morphological changes characterized primarily by cellular rounding. All of these effects were blocked to a significant degree if cells were preincubated with mevastatin in the presence of $1 \mathrm{mM}$ mevalonate (Fig. 6). Pretreatment with mevalonate alone did not alter the basal or agonist-stimulated phosphorylation of FAK or paxillin (data not shown).

Although recent findings have demonstrated that the low-molecular-weight $G$ protein Rho may play a role in mediation of the tyrosine phosphorylation of FAK in fibroblasts (Rankin et al., 1994; Flinn and Ridley, 1996), no such evidence was obtained for SH-SY5Y cells. Thus, a 48-h preincubation of SH-SY5Y cells with $12.5 \mu \mathrm{g} / \mathrm{ml} \mathrm{C} 3$ exoenzyme (a protein isolated from Clostridium botulinum that selectively ADP-ribosylates and inactivates Rho proteins ) had no significant
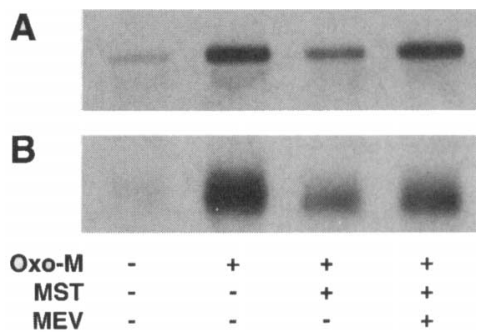

FIG. 6. Mevastatin (MST) pretreatment inhibits the muscarinic receptor-mediated tyrosine phosphorylation of FAK and paxillin: attenuation of the inhibitory effects of MST by inclusion of mevalonate (MEV). Confluent cultures of SH-SY5Y cells were pretreated for $24 \mathrm{~h}$ with either vehicle or $10 \mu \mathrm{M}$ MST with or without $1 \mathrm{mM}$ MEV before addition of either buffer or Oxo-M (final concentration, $1 \mathrm{mM}$ ) for $10 \mathrm{~min}$. Following agonist exposure, FAK (A) or paxillin (B) immune complexes were isolated, subjected to SDS-PAGE, transferred to PVDF, and immunoblotted for phosphotyrosine as described in the legend to Fig. 1. The blots in $A$ and $B$ are representative of three and two independent experiments, respectively.

effect on the Oxo-M-stimulated tyrosine phosphorylation of FAK (Fig. 7). Subsequent in vitro measurements of ADP-ribosylation of Rho proteins in lysates from C3 exoenzyme-pretreated SH-SY5Y cells revealed an $\sim 35 \%$ decrease in ${ }^{32} \mathrm{P}$ incorporation compared with vehicle-pretreated cells.

\section{Wortmannin and LY-294002 attenuate the agonist-stimulated tyrosine phosphorylation of FAK and paxillin and selectively decrease ${ }^{32} \mathrm{P}$ labeling of PI(4)P}

Phosphoinositides may play a key role in the maintenance of the cytoskeleton (Lassing and Lindberg, 1985; Yu et al., 1990; Fukami et al., 1992; Hartwig et al., 1995). Given that an intact cytoskeleton is a prerequisite for the agonist-stimulated tyrosine phosphorylation of FAK and paxillin, a direct role for the inositol lipids in these signaling events was investigated by examining the effects of two structurally unrelated inhibitors of phosphoinositide synthesis. Inclusion of low concentrations of either wortmannin $(\leq 100 \mathrm{n} M)$ or LY-294002 ( $\leq 10 \mu M)$, conditions under which PI 3-kinase activity is markedly inhibited in SH-SY5Y cells (>95\% inhibition with $100 \mathrm{nM}$

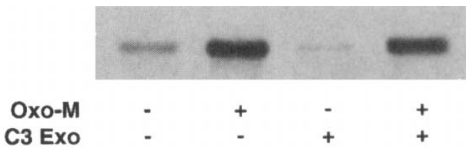

FIG. 7. C3 exoenzyme (C3 Exo) pretreatment fails to inhibit Oxo-M-induced FAK phosphorylation. Confluent cultures of $\mathrm{SH}$ SY5Y cells were preincubated for $48 \mathrm{~h}$ with either vehicle or 12.5 $\mu \mathrm{g} / \mathrm{ml}$ C3 Exo in DMEM containing $10 \%$ FCS before a $10-\mathrm{min}$ exposure to $1 \mathrm{mM}$ Oxo-M. Following agonist treatment, FAK immune complexes were isolated, subjected to SDS-PAGE, transferred to PVDF, and immunoblotted for phosphotyrosine as described in the legend to Fig. 1. The blot shown is representative of three independent experiments. 
wortmannin and $>60 \%$ inhibition with $10 \mu \mathrm{M}$ LY294002), had no effect on the agonist-stimulated tyrosine phosphorylation of either FAK or paxillin. However, pretreatment of SH-SY5Y cells with higher concentrations of either inhibitor, conditions under which PI 4-kinase activity is reported to be inhibited (Nakanishi et al., 1995; Downing et al., 1996; Meyers and Cantley, 1997), resulted in complete inhibition of the tyrosine phosphorylation of FAK ( $\mathrm{IC}_{50}$ values for wortmannin and LY-294002, $478 \mathrm{n} M$ and $23 \mu M$, respectively; Figs. 8A and $\mathrm{B}$ and $9 \mathrm{~A}$ and $\mathrm{B}$ ). In parallel experiments, both wortmannin and LY-294002 also inhibited the tyrosine phosphorylation of paxillin with dose-inhibition relationships similar to those observed for FAK (Figs. 8C and 9C).

Consistent with the ability of these agents to inhibit PI 4-kinase activity, pretreatment of SH-SY5Y cells with either $10 \mu M$ wortmannin or $100 \mu M$ LY -294002 resulted in a selective decrease in the basal ${ }^{32} \mathrm{P}$ labeling of PI(4)P by $79 \pm 2$ and $60 \pm 4 \%$, respectively ( $\mathrm{n}$ $=3$; Fig. 10). Neither inhibitor had any significant effect on the basal labeling of PA, PI, or PI 4,5-bisphosphate $\left[\mathrm{PI}(4,5) \mathrm{P}_{2}\right]$. Following addition of Oxo-M to ${ }^{32} \mathrm{P}$-prelabeled cells, there was a marked increase in $\left[{ }^{32} \mathrm{P}\right] \mathrm{PA}$ and $\left[{ }^{32} \mathrm{P}\right] \mathrm{PI}$ labeling, whereas the radioactivity incorporated into $\mathrm{PI}(4) \mathrm{P}$ and $\mathrm{PI}(4,5) \mathrm{P}_{2}$ was de-
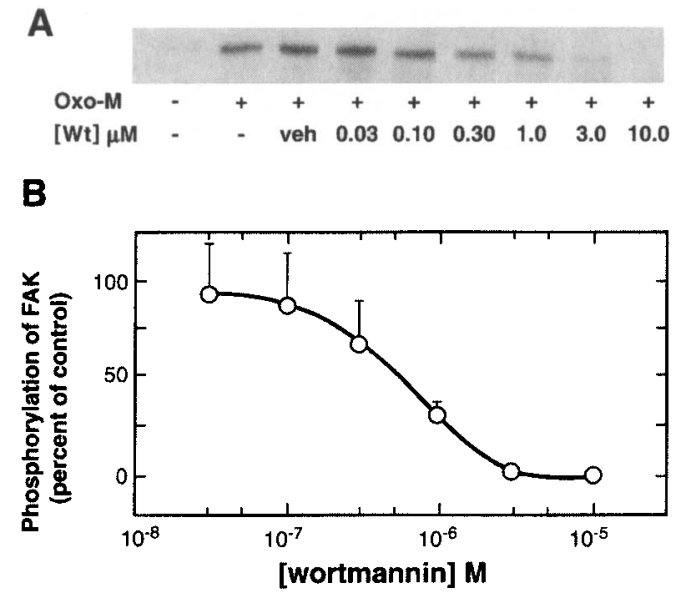

C

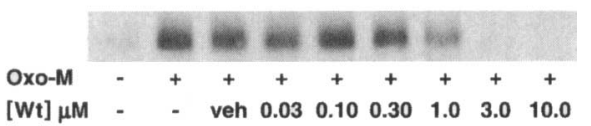

FIG. 8. Wortmannin (Wt) pretreatment inhibits the Oxo-M-stimulated tyrosine phosphorylation of FAK and paxillin. Confluent cultures of SH-SY5Y cells were pretreated for $10 \mathrm{~min}$ with either vehicle (veh; $0.2 \%$ dimethyl sulfoxide) or Wt at the indicated concentrations before addition of Oxo-M (final concentration, 1 $\mathrm{mM}$ ) for an additional $10 \mathrm{~min}$. Following treatment, FAK (A) or paxillin (C) immune complexes were isolated, subjected to SDSPAGE, transferred to PVDF, and immunoblotted for phosphotyrosine as described in the legend to Fig. 1. The blots in $A$ and $C$ are representative of three and two independent experiments, respectively. B: Densitometric analysis of the Oxo-M-stimulated tyrosine phosphorylation of FAK obtained from three independent experiments.
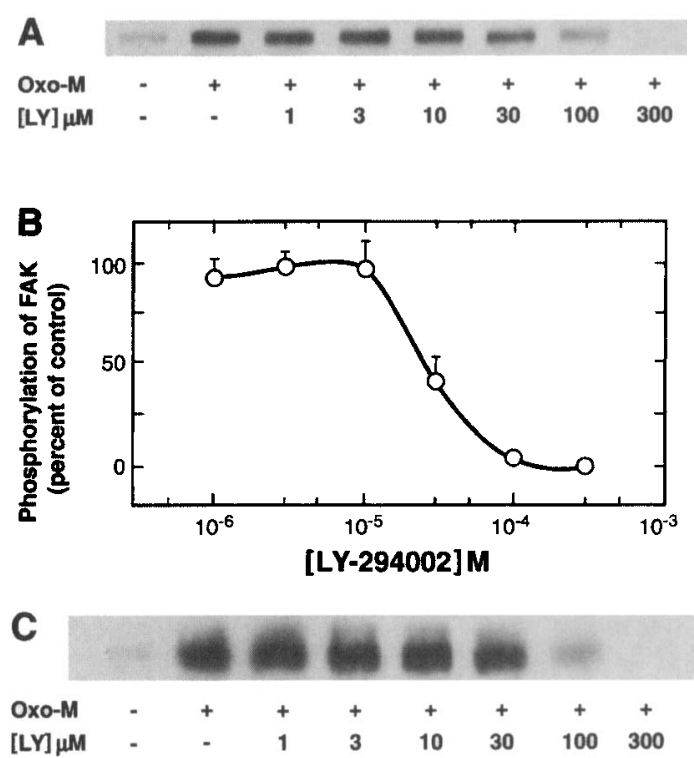

FIG. 9. LY-294002 (LY) pretreatment attenuates the Oxo-Minduced tyrosine phosphorylation of FAK and paxillin. Confluent cultures of SH-SY5Y cells were pretreated for $10 \mathrm{~min}$ with the indicated concentrations of LY before addition of OxO-M (final concentration, $1 \mathrm{mM}$ ) for an additional $10 \mathrm{~min}$. Following treatment, FAK (A) or paxillin (C) immune complexes were isolated, subjected to SDS-PAGE, transferred to PVDF, and immunoblotted for phosphotyrosine as described in the legend to Fig. 1. The blots in $A$ and $C$ are representative of three and two independent experiments, respectively. B: Densitometric analysis of the OxoM-stimulated tyrosine phosphorylation of FAK obtained from three independent experiments.

creased (Fig. 10). Preincubation of ${ }^{32} \mathrm{P}$-labeled cells with either wortmannin or LY-294002 substantially reduced the agonist-mediated increases in the labeling of PA and PI and resulted in further decreases in the labeling of $\mathrm{PI}(4) \mathrm{P}$ and $\mathrm{PI}(4,5) \mathrm{P}_{2}$ (Fig. 10). As a consequence of their ability to block the resynthesis of PI(4) P [ and PI (4,5) $\mathrm{P}_{2}$ ], both wortmannin and LY294002 were shown to inhibit mAChR-stimulated phosphoinositide hydrolysis, as monitored by the production of ${ }^{3} \mathrm{H}$-inositol phosphates from $\left[{ }^{3} \mathrm{H}\right]$ inositolprelabeled cells, with $\mathrm{IC}_{50}$ values of $537 \mathrm{n} M$ and 32 $\mu M$, respectively.

\section{DISCUSSION}

In this study, we demonstrate that agonist occupancy of $m_{3} \mathrm{mAChRs}$ in SH-SY5Y neuroblastoma cells results in an increased tyrosine phosphorylation of FAK and paxillin, two proteins that have recently been implicated in a signal transduction pathway linked to neurite outgrowth (Bianchi et al., 1995; Maroney et al., 1995; Leventhal and Feldman, 1996; Leventhal et al., 1997). Although two previous studies have reported that stimulation of mAChRs transfected into either fibroblasts (Gutkind and Robbins, 1992) or Chinese hamster ovary cells (Savage et al., 1995) can elicit an 

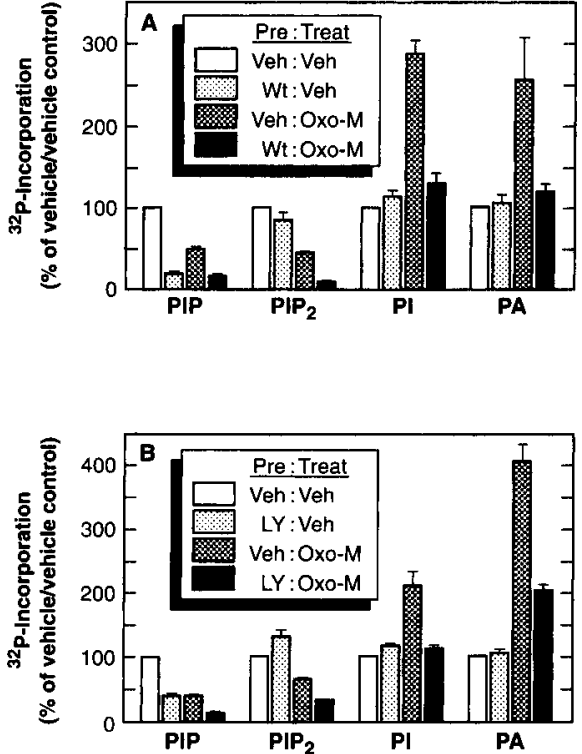

FIG. 10. Wortmannin (Wt) and LY-294002 (LY) selectively decrease the incorporation of ${ }^{32} \mathrm{P}_{i}$ into PI(4)P (PIP). Confluent cultures of $\mathrm{SH}-\mathrm{SY} 5 \mathrm{Y}$ cells were prelabeled with ${ }^{32} \mathrm{P}_{\mathrm{i}}$ for $2 \mathrm{~h}$. Unincorporated label was washed away, and the cells were pretreated (Pre) for 10 min with vehicle (Veh; $0.2 \%$ dimethyl sulfoxide), 10 $\mu M \mathrm{Wt}(\mathbf{A})$, or $100 \mu M L Y(\mathbf{B})$ before treatment (Treat) with either veh or OxO-M (final concentration, $1 \mathrm{mM}$ ) for an additional 10 min. Reactions were terminated by addition of TCA, lipids were extracted and separated, and radioactivity was quantified as described in Materials and Methods. Data are mean \pm SEM (bars) values for three independent experiments with each inhibitor and are expressed as percentages of the veh/veh control value. $\mathrm{PIP}_{2}, \mathrm{PI}(4,5) \mathrm{P}_{2}$.

increase in the tyrosine phosphorylation of FAK, the present study is the first demonstration of such for an endogenous $\mathrm{mAChR}$ in a neural cell line. In addition, the mAChR-mediated tyrosine phosphorylation of FAK and paxillin was observed in both undifferentiated and retinoic acid-differentiated SH-SY5Y cells. The mAChR-mediated tyrosine phosphorylation of FAK and paxillin in SH-SY5Y cells is independent of integrin interactions with the ECM, as phosphorylation of both proteins could be readily demonstrated when cells were maintained in suspension (Fig. 3). This observation is in contrast to previous reports demonstrating that, following activation of integrin receptors, detachment of cells from the ECM results in the rapid dephosphorylation of FAK and paxillin (Kornberg et al., 1992; Leventhal and Feldman, 1996). However, our results are in agreement with a recent study in which an enhanced phosphorylation of FAK was observed in response to addition of either LPA or active Rho protein to suspensions of (scrape-loaded) Swiss 3 T3 fibroblasts (Flinn and Ridley, 1996). Thus, it appears that cell attachment is not invariably a prerequisite for the increased tyrosine phosphorylation of FAK and paxillin and that the signaling events that initiate this phosphorylation cascade following $\mathrm{mAChR}$ acti- vation in SH-SY5Y cells can occur in an environment devoid of focal contacts with the ECM.

There is some uncertainty regarding the role that phosphoinositide-derived second messengers, e.g., $\mathrm{IP}_{3}$ and DAG, may play in mediating the enhanced tyrosine phosphorylation of FAK following agonist occupancy of receptors coupled to PIC activation. For example, whereas evidence suggesting the involvement of either PKC or $\mathrm{Ca}^{2+}$ has been obtained (Leeb-Lundberg et al., 1994; Savage et al., 1995), the results from other studies are inconsistent with these observations (Sinnet-Smith et al., 1993; Seufferlein and Rozengurt, $1994 b$ ). Our results indicate that, in SH-SY5Y cells, the production of phosphoinositide-derived second messengers is not a prerequisite for the enhanced tyrosine phosphorylation of FAK elicited by $\mathrm{mAChR}$ stimulation. The evidence for this conclusion is threefold. First, neither the inhibition of PKC by Ro 31-8220 nor its depletion from cells by chronic PMA pretreatment decreased the extent of tyrosine phosphorylation of FAK in response to $\mathrm{mAChR}$ activation. Second, neither depletion of intracellular $\mathrm{Ca}^{2+}$ stores with thapsigargin nor blockade of $\mathrm{Ca}^{2+}$ entry with $\mathrm{Ni}^{2+}$ attenuated the phosphorylation of FAK. Third, no evidence was obtained for the involvement of other putative downstream messenger molecules such as PA or LPA, given that inhibitors of DAG kinase and phospholipase $\mathrm{A}_{2}$ had no effect on the agonist-stimulated phosphorylation of FAK. The absence of a requirement for PKC activation in the $\mathrm{mAChR}$-mediated tyrosine phosphorylation of FAK is in contrast to the previously demonstrated role for PKC in the stimulation of the mitogenactivated protein kinase (MAPK) pathway elicited by activation of the same receptors in these cells (Offermanns et al., 1993). This observation is of significance because the tyrosine phosphorylation of FAK has been linked to activation of the Ras/MAPK cascade via the association of FAK with the adapter protein GRB-2 (Schlaepfer et al., 1994). However, in SHSY5Y cells, the mAChR-mediated activation of FAK and that of MAPK appear to be parallel, but independent, events.

A characteristic feature of the tyrosine phosphorylation of FAK elicited in response to addition of a diverse range of stimuli is its susceptibility to addition of cytochalasin $D$ with the attendant disruption of the actin cytoskeleton. The increases in the tyrosine phosphorylation of FAK and paxillin resulting from $\mathrm{mAChR}$ activation in SH-SY5Y cells were similarly prevented by inclusion of cytochalasin D (Fig. 5). Cytochalasin D sensitivity was also observed for $\mathrm{mAChR}$-mediated increases in the tyrosine phosphorylation of FAK elicited in SH-SY5Y cells maintained in suspension (data not shown). In contrast, other consequences of $\mathrm{mAChR}$ activation in these cells, such as receptor internalization and MAPK activation, were unaffected by cytochalasin D. The conclusion that an intact actin cytoskeleton is required for FAK phosphorylation was further substantiated by the observation that pretreat- 
ment of the cells with mevastatin, an agent known to destabilize actin microfilaments, also attenuated the agonist-stimulated tyrosine phosphorylation of FAK and paxillin. The inhibitory effects of mevastatin could largely be prevented by inclusion of mevalonate, a precursor molecule required for the isoprenylation of various proteins, including some low-molecularweight $\mathrm{G}$ proteins implicated in the regulation of the cytoskeleton. In this context, recent studies indicate that in fibroblasts, the low-molecular-weight $G$ protein p21Rho may play a pivotal role in mediating the tyrosine phosphorylation of FAK and paxillin (Rankin et al., 1994; Flinn and Ridley, 1996). This may not be the case for the mAChR-mediated tyrosine phosphorylation of FAK in SH-SY5Y neuroblastoma cells because pretreatment with C3 exoenzyme failed to prevent the agonist-induced increase in the tyrosine phosphorylation of FAK. However, as only a partial ADPribosylation of Rho proteins in SH-SY5Y cells was observed, any such conclusion must remain tentative. Recently it has been shown that $\mathrm{mAChR}$-mediated actin stress fiber formation in transfected Chinese hamster ovary cells is also insensitive to C3 exoenzyme (Williams et al., 1996). Taken collectively, these results suggest that if a low-molecular-weight $G$ protein is involved in the mAChR-mediated alterations of the cytoskeleton and signaling events that lead to the tyrosine phosphorylation of FAK, it may not invariably be Rho.

The actin cytoskeleton is known to be markedly influenced by phosphoinositides. For example, these lipids modulate the functions of several actin binding proteins (Lassing and Lindberg, 1985; Yu et al., 1990; Fukami et al., 1992) and can induce actin filament uncapping, thereby facilitating actin polymerization (Hartwig et al., 1995). A major finding to emanate from the present study is that the synthesis of polyphosphoinositides, specifically that of PI(4)P, is required for the increased tyrosine phosphorylation of FAK and paxillin in response to $\mathrm{mAChR}$ activation. Pretreatment of SH-SY5Y cells with concentrations of either wortmannin or LY-294002 that inhibit the synthesis of PI(4)P resulted in a marked attenuation of the phosphorylation of FAK and paxillin (see Figs. 8 and 9). In contrast, no inhibition of phosphorylation was observed at concentrations of these inhibitors ( $\leq 100 \mathrm{n} M$ for wortmannin and $\leq 10 \mu M$ for LY294002 ) that are more specific for inhibition of PI 3kinase (Yano et al., 1995). In this context, it should be noted that inclusion of either $30 \mathrm{n} M$ wortmannin or $10 \mu M$ LY-294002 has been reported to inhibit the enhanced tyrosine phosphorylation of FAK in plateletderived growth factor-stimulated fibroblasts by $65-$ $100 \%$, a result consistent with the involvement of PI 3-kinase (Rankin et al., 1996). However, in the same study, these inhibitor concentrations were without effect on the tyrosine phosphorylation of FAK induced by activation of bombesin receptors (which, like mAChRs, couple to PIC via $G_{q}$ ). Although both wort- mannin and LY-294002 have also been demonstrated to inhibit activity of myosin light chain kinase (MLCK) (Yano et al., 1995), a key enzyme involved in the regulation of actin/myosin interactions and cell contractility (see Burridge and Chrzanowska-Wodnicka, 1996), a role for this enzyme in the mAChRmediated tyrosine phosphorylation of FAK and paxillin appears unlikely for three reasons. First, although MLCK is a $\mathrm{Ca}^{2+}$-dependent enzyme, there is no such $\mathrm{Ca}^{2+}$ requirement for the phosphorylation of $\mathrm{FAK}$ and paxillin (see Fig. 4C). Second, pretreatment of SHSY5Y cells with $10 \mu M$ ML-7, a known inhibitor of MLCK, was without effect on the phosphorylation of FAK (data not shown). Third, whereas pretreatment of SH-SY5Y cells with $100 \mu M$ LY-294002 resulted in a $>95 \%$ inhibition of the tyrosine phosphorylation of FAK and paxillin, only a small inhibitory effect on MLCK is observed at this concentration (Yano et al., 1995). An additional possibility, i.e., that wortmannin directly inhibits the autokinase activity of FAK, also appears unlikely given that other tyrosine kinases, e.g., Src or MEK, are not inhibited by wortmannin (Brunn et al., 1996). In agreement with their reported ability to inhibit PI 4-kinase activity (Nakanishi et al., 1995; Downing et al., 1996; Meyers and Cantley, 1997), addition of either wortmannin or LY-294002 to SHSY5Y cells resulted in a selective inhibition of PI(4)P synthesis, as demonstrated by a decreased ${ }^{32} \mathrm{P}$ labeling (Fig. 10). In the presence of Oxo-M, which when added alone reduces $\mathrm{PI}(4) \mathrm{P}$ and $\mathrm{PI}(4,5) \mathrm{P}_{2}$ labeling, the inclusion of either inhibitor resulted in a further reduction in polyphosphoinositide labeling. As anticipated, inhibition of polyphosphoinositide synthesis by wortmannin or LY-294002 resulted in a marked reduction in the extent of mAChR-stimulated inositol lipid hydrolysis, as monitored by the release of radiolabeled inositol phosphates. From dose-inhibition studies, the latter parameter was found to be highly correlated with the extent of inhibition of the tyrosine phosphorylation of FAK for both wortmannin $(r=0.995, p<0.001)$ and LY-294002 ( $r=0.976, p<0.01)$. Taken collectively, these results suggest that wortmannin and LY294002 deplete the cellular content of phosphoinositides. A consequence of this may be that the normal maintenance of the actin cytoskeleton by these lipids is compromised, thereby depriving FAK of the required environment in which it can autophosphorylate and become fully activated.

In summary, the present results indicate that on agonist occupancy of mAChRs in SH-SY5Y cells, there is a marked increase in the tyrosine phosphorylation of FAK and paxillin. Increases in phosphorylation occur independently of the production of phosphoinositidederived second messengers but require the continuous synthesis of inositol lipids. The latter may reflect a role for these lipids in the maintenance of an intact actin cytoskeleton, which is a prerequisite for the increased tyrosine phosphorylation of FAK and paxillin. 
Acknowledgment: The authors wish to thank Dr. Anne Heacock for helpful comments and Ms. Jo Ann Kelsch and Tim Desmond for preparation of the manuscript. This work was supported by grant NS 23831 from the National Institutes of Health and grant MH 46252 from the National Institute of Mental Health (to S.K.F.). D.A.L. was supported by training grant GM 07767 from the National Institutes of Health.

\section{REFERENCES}

Bellis S. L., Miller J. T., and Turner C. E. (1995) Characterization of tyrosine phosphorylation of paxillin in vitro by focal adhesion kinase. J. Biol. Chem. 270, 17437-17441.

Bianchi L., Arcangeli A., Bartolini P., Mugnai G., Wanke E., and Olivotto M. (1995) An inward rectifier $\mathrm{K}^{+}$current modulates in neuroblastoma cells the tyrosine phosphorylation of the pp $125^{\mathrm{FAK}}$ and associated proteins: role in neuritogenesis. Biochem. Biophys. Res. Commun. 210, 823-829.

Bifulco M., Laezza C., Aloj S. M., and Garbi C. (1993) Mevalonate controls cytoskeleton organization and cell morphology in thyroid epithelial cells. J. Cell. Physiol. 155, 340-348.

Bokoch G. M. and Prossnitz V. (1992) Isoprenoid metabolism is required for stimulation of the respiratory burst oxidase of $\mathrm{HL}$ 60 cells. J. Clin. Invest. 89, 402-408.

Brunn G. J., Williams J., Sabers C., Wiederrecht G., Lawrence J. C. Jr., and Abraham R. T. (1996) Direct inhibition of the signaling functions of the mammalian target of rapamycin by the phosphoinositide 3-kinase inhibitors, wortmannin and LY294002. $E M B O ~ J .15,5256-5267$.

Burridge K. and Chrzanowska-Wodnicka M. (1996) Focal adhesions, contractility, and signaling. Annu. Rev. Cell Dev. Biol. $12,463-518$.

Burridge K., Turner C. E., and Romer L. H. (1992) Tyrosine phosphorylation of paxillin and pp125 ${ }^{\mathrm{FAK}}$ accompanies cell adhesion to extracellular matrix: a role in cytoskeletal assembly. $J$. Cell Biol. 119, 893-903.

Derkinderen P., Toutant M., Burgaya F., Le Bert M., Siciliano J. C., de Franciscis V., Gelman M., and Girault J.-A. (1996) Regulation of a neuronal form of focal adhesion kinase by anandamide. Science 273, 1719-1722.

Downing G. J., Kim S., Nakanishi S., Catt K. J., and Balla T. (1996) Characterization of a soluble adrenal phosphatidylinositol 4kinase reveals wortmannin sensitivity of type III phosphatidylinositol kinases. Biochemistry 35, 3587-3594.

Fenton R. G., Kung H., Longo D. L., and Smith M. R. (1992) Regulation of intracellular actin polymerization by prenylated cellular proteins. J. Cell Biol. 117, 347-356.

Fisher S. K. and Agranoff B. W. (1980) Calcium and the muscarinic synaptosomal phospholipid labeling effect. J. Neurochem. 34, $1231-1240$.

Fisher S. K., Figueiredo J. C., and Bartus R. T. (1984) Differential stimulation of inositol phospholipid turnover in brain by analogs of oxotremorine. J. Neurochem. 43, 1171-1179.

Fisher S. K., Slowiejko D. M., and McEwen E. L. (1994) A rapid attenuation of muscarinic agonist stimulated phosphoinositide hydrolysis precedes receptor sequestration in human SH-SY5Y neuroblastoma cells. Neurochem. Res. 19, 549-554.

Flinn H. M. and Ridley A. J. (1996) Rho stimulates tyrosine phosphorylation of focal adhesion kinase, p130 and paxillin. J. Cell Sci. 109, 1133-1141.

Fukami K., Furuhashi K., Inagaki M., Endo T., Hatano S., and Takenawa T. (1992) Requirement of phosphatidylinositol 4,5bisphosphate for $\alpha$-actinin function. Nature 359, 150-152.

Grant S. G. N., Karl K. A., Kiebler M. A., and Kandel E. R. (1995) Focal adhesion kinase in the brain: novel subcellular localization and specific regulation by fyn tyrosine kinase in mutant mice. Genes Dev. 9, 1909-1921.

Guan J.-L. and Shalloway D. (1992) Regulation of focal adhesionassociated protein tyrosine kinase by both cellular adhesion and oncogenic transformation. Nature 358, 690-692.
Gutkind J. S. and Robbins K. C. (1992) Activation of transforming $\mathrm{G}$ protein-coupled receptors induces rapid tyrosine phosphorylation of cellular proteins, including $\mathrm{p} 125^{\mathrm{FAK}}$ and the p130 vsrc substrate. Biochem. Biophys. Res. Commun. 188, 155-161.

Hanks S. K., Calalb M. B., Harper M. C., and Patel S. K. (1992) Focal adhesion protein-tyrosine kinase phosphorylated in response to cell attachment to fibronectin. Proc. Natl. Acad. Sci. USA 89, 8487-8491.

Hartwig J. H., Bokoch G. M., Carpenter C. L., Janmey P. A., Taylor L. A., Toker A., and Stossel T. P. (1995) Thrombin receptor ligation and activated rac uncap actin filament barbed ends through phosphoinositide synthesis in permeabilized human platelets. Cell 82, 643-653.

Honegger P. and Richelson E. (1976) Biochemical differentiation of mechanically dissociated mammalian brain in aggregating cell culture. Brain Res. 109, 335-354.

Kornberg L., Earp H. S., Parsons J. T., Schaller M., and Juliano R. L. (1992) Cell adhesion or integrin clustering increases phosphorylation of a focal adhesion-associated tyrosine kinase. J. Biol. Chem. 267, 23439-23442.

Laemmli U. K. (1970) Cleavage of structural proteins during the assembly of the head of bacteriophage T4. Nature 227, 680 685 .

Lassing I. and Lindberg U. (1985) Specific interaction between phosphatidylinositol 4,5-bisphosphate and profilactin. Nature 314, $472-474$

Lavie Y. and Agranoff B. W. (1996) Carbachol inhibits insulinstimulated phosphatidylinositol 3-kinase activity in SH-SY5Y neuroblastoma cells. J. Neurochem. 67, 1245-1251.

Leeb-Lundberg L. M., Song X.-H., and Mathis S. A. (1994) Focal adhesion-associated proteins $\mathrm{p} 125^{\mathrm{FAK}}$ and paxillin are substrates for bradykinin-stimulated tyrosine phosphorylation in Swiss 3T3 cells. J. Biol. Chem. 269, 24328-24334.

Leventhal P. S. and Feldman E. L. (1996) Tyrosine phosphorylation and enhanced expression of paxillin during neuronal differentiation in vitro. J. Biol. Chem. 271, 5957-5960.

Leventhal P. S., Shelden E. A., Kim B., and Feldman E. L. (1997) Tyrosine phosphorylation of paxillin and focal adhesion kinase during insulin-like growth factor-I-stimulated lamellipodial advance. J. Biol. Chem. 272, 5214-5218.

Linseman D. A., McEwen E. L., and Fisher S. K. (1997) Activation of muscarinic receptors in SH-SY5Y neuroblastoma cells elicits a wortmannin-sensitive tyrosine phosphorylation of focal adhesion kinase. (Abstr.) Life Sci. 60, 1183.

Maroney A. C., Lipfert L., Forbes M. E., Glicksman M. A., Neff N. T., Siman R., and Dionne C. A. (1995) K-252a induces tyrosine phosphorylation of the focal adhesion kinase and neurite outgrowth in human neuroblastoma SH-SY5Y cells. J. Neurochem. $64,540-549$.

Méyers R. and Cantley L. C. (1997) Cloning and characterization of a wortmannin-sensitive human phosphatidylinositol 4-kinase. J. Biol. Chem. 272, 4383-4390.

Nakanishi S., Catt K. J., and Balla T. (1995) A wortmannin-sensitive phosphatidylinositol 4-kinase that regulates hormone-sensitive pools of inositol phospholipids. Proc. Natl. Acad. Sci. USA 92, $5317-5321$.

Offermanns S., Bombien E., and Schultz G. (1993) Stimulation of tyrosine phosphorylation and mitogen-activated-protein (MAP) kinase activity in human SH-SY5Y neuroblastoma cells by carbachol. Biochem. J. 294, 545-550.

Rankin S., Morii N., Narumiya S., and Rozengurt E. (1994) Botulinum C3 exoenzyme blocks the tyrosine phosphorylation of p $125^{\mathrm{FAK}}$ and paxillin induced by bombesin and endothelin. FEBS Lett. 354, 315-319.

Rankin S., Hooshmand-Rad R., Claesson-Welsh L., and Rozengurt E. (1996) Requirement for phosphatidylinositol 3'-kinase activity in platelet-derived growth factor-stimulated tyrosine phosphorylation of p125 focal adhesion kinase and paxillin. J. Biol. Chem. 271, 7829-7834.

Rösner H., Vacun G., and Rebhan M. (1995) Muscarinic receptormediated induction of actin-driven lamellar protrusions in neu- 
roblastoma cell somata and growth cones. Involvement of protein kinase C. Eur. J. Cell Biol. 66, 324-334.

Savage S. A., Kohn E. C., and Spoonster J. S. ( 1995) Focal adhesion kinase (FAK) regulation in the muscarinic (subtype 5) acetylcholine receptor ( $\mathrm{m5AChR}$ ) tumor suppressor model. (Abstr.) Proc. Annu. Meet. Am. Assoc. Cancer Res. 36, A311.

Schaller M. D. and Parsons J. T. (1995) pp $125^{\text {FAK }_{\text {-dependent tyro- }}}$ sine phosphorylation of paxillin creates a high-affinity binding site for crk. Mol. Cell. Biol. 15, 2635-2645.

Schaller M. D., Borgman C. A., Cobb B. S., Vines R. R., Reynolds A. B., and Parsons J. T. (1992) pp125 $5^{\mathrm{FAK}}$, a structurally distinctive protein-tyrosine kinase associated with focal adhesions. Proc. Natl. Acad. Sci. USA 89, 5192-5196.

Schlaepfer D. D., Hanks S. K., Hunter T., and van der Geer P. (1994) Integrin-mediated signal transduction linked to Ras pathway by GRB2 binding to focal adhesion kinase. Nature 372, 786-791.

Seufferlein T. and Rozengurt E. (1994a) Sphingosine induces p125 $5^{\mathrm{FAK}}$ and paxillin tyrosine phosphorylation, actin stress fiber formation, and focal contact assembly in Swiss 3T3 cells. $J$. Biol. Chem. 269, 27610-27617.

Seufferlein T. and Rozengurt E. (1994b) Lysophosphatidic acid stimulates tyrosine phosphorylation of focal adhesion kinase, paxillin, and p130. Signaling pathways and cross-talk with platelet-derived growth factor. J. Biol. Chem. 269, 9345-9351.

Sinnett-Smith J., Zachary I., Valverde A. M., and Rozengurt E. (1993) Bombesin stimulation of p125 focal adhesion kinase tyrosine phosphorylation. Role of protein kinase $\mathrm{C}, \mathrm{Ca}^{2+}$ mobilization, and the actin cytoskeleton. J. Biol. Chem. 268, 14261 14268.

Slowiejko D. M., Levey A. I., and Fisher S. K. (1994) Sequestration of muscarinic cholinergic receptors in permeabilized neuroblastoma cells. J. Neurochem. 62, 1795-1803.

Slowiejko D. M., McEwen E. L., Ernst S. A., and Fisher S. K. (1996) Muscarinic receptor sequestration in SH-SY5Y neuro- blastoma cells is inhibited when clathrin distribution is perturbed. J. Neurochem. 66, 186-196.

Thompson A. K. and Fisher S. K. (1990) Relationship between agonist-induced muscarinic receptor loss and desensitization of stimulated phosphoinositide turnover in two neuroblastomas: methodological considerations. J. Pharmacol. Exp. Ther. 252, $744-752$.

Wahl M. I., Daniel T. O., and Carpenter G. (1988) Antiphosphotyrosine recovery of phospholipase $C$ activity after EGF treatment of A-431 cells. Science 241, 968-970.

Williams C. L., Phelps S. H., and Porter R. A. (1996) ADP-ribosylation of rho-protein does not inhibit stress fiber formation induced by $\mathrm{m}(1)$ or $\mathrm{m}(3)$ muscarinic acetylcholine-receptor activation. (Abstr.) Mol. Biol. Cell 7 (Suppl.), 3200.

Wojcikiewicz R. J. H., Tobin A. B., and Nahorski S. R. (1994) Muscarinic receptor-mediated inositol 1,4,5-trisphosphate formation in SH-SY5Y neuroblastoma cells is regulated acutely by cytosolic $\mathrm{Ca}^{2+}$ and by rapid desensitization. J. Neurochem. 63, $177-185$.

Yamamoto M., Marui N., Sakai T., Morii N., Kozaki S., Ikai K., Imamura S., and Narumiya S. (1993) ADP-ribosylation of the rhoA gene product by botulinum C3 exoenzyme causes Swiss $3 T 3$ cells to accumulate in the $\mathrm{G}_{1}$ phase of the cell cycle. Oncogene 8, 1449-1455.

Yano H., Agatsuma T., Nakanishi S., Saitoh Y., Fukui Y., Nonomura Y., and Matsuda Y. (1995) Biochemical and pharmacological studies with KT7692 and LY294002 on the role of phosphatidylinositol 3-kinase in Fc $\in$ RI-mediated signal transduction. Biochem. J. 312, 145-150.

Yu F.-X., Johnston P. A., Südhof T. C., and Yin H. L. (1990) gCap39, a calcium ion- and polyphosphoinositide-regulated actin capping protein. Science 250, 1413-1415.

Zhang C., Lambert M. P., Bunch C., Barber K., Wade W. S., Krafft G. A., and Klein W. L. (1994) Focal adhesion kinase expressed by nerve cell lines shows increased tyrosine phosphorylation in response to Alzheimer's A $\beta$ peptide. J. Biol. Chem. 269, $25247-25250$. 